\title{
Simulation Study of Energetic Ion Driven Instabilities near the Lower Hybrid Resonance Frequency in a Plasma with Increasing Density ${ }^{*)}$
}

\author{
Mieko TOIDA $^{1)}$, Hiroe IGAMI ${ }^{1)}$, Kenji SAITO ${ }^{1)}$, Tsuyoshi AKIYAMA ${ }^{1,2)}$, \\ Shuji KAMIO ${ }^{1)}$ and Ryosuke SEKI ${ }^{1,2)}$ \\ 1) National Institute for Fusion Science, Toki, Gifu 509-5292, Japan \\ ${ }^{2}$ SOKENDAI (The Graduate University for Advanced Studies), Department of Fusion Science, Toki, \\ Gifu 509-5292, Japan
}

(Received 9 January 2019 / Accepted 26 April 2019)

\begin{abstract}
Using a one-dimensional electromagnetic particle code which simulates self-consistently the full ion and electron dynamics, we study instabilities driven by energetic ions injected continuously in a plasma where the density and the lower-hybrid resonance frequency $\omega_{\mathrm{LH}}$ increase with time. The simulation shows that the ion cyclotron harmonic wave with $\omega \simeq l \Omega_{\mathrm{i}}$, where $l$ is an integer and $\Omega_{\mathrm{i}}$ is the ion cyclotron frequency, is excited when $\omega_{\mathrm{LH}}$ becomes close to $l \Omega_{\mathrm{i}}$. When $\omega_{\mathrm{LH}}$ is greater than $l \Omega_{\mathrm{i}}$, this wave couples with the ion Bernstein mode that has the dispersion curve connecting to $\omega_{\mathrm{LH}}$. It is also found that as a result of the instabilities and the wave-wave coupling, the stair-like frequency chirping with the riser $\Omega_{\mathrm{i}}$ appears in the magnetic fluctuations in the frequency range of $\omega_{\mathrm{LH}}$. The frequency chirping has characteristics similar to the frequency chirping observed in the RF radiations at the plasma start-up phase of the LHD experiments.
\end{abstract}

(c) 2019 The Japan Society of Plasma Science and Nuclear Fusion Research

Keywords: instabilities driven by energetic ions, PIC simulation, lower hybrid resonance frequency, ion cyclotron wave, ion Bernstein wave, Large Helical Device

DOI: $10.1585 / \mathrm{pfr} .14 .3401112$

\section{Introduction}

Waves with frequencies near the lower hybrid resonance frequency $\omega_{\mathrm{LH}}$ propagating perpendicular to the magnetic field can be excited by energetic ion beams across the magnetic field when the beam speed is smaller than the Alfvén speed [1-3]. Here, $\omega_{\mathrm{LH}}$ is defined as

$$
\begin{aligned}
\omega_{\mathrm{LH}}^{2} & =\Omega_{\mathrm{i}} \Omega_{\mathrm{e}}\left(1+\Omega_{\mathrm{i}}^{2} / \omega_{\mathrm{pi}}^{2}\right) /\left(1+\Omega_{\mathrm{e}}^{2} / \omega_{\mathrm{pe}}^{2}\right) \\
& =\left(\Omega_{\mathrm{i}}^{2}+\omega_{\mathrm{pi}}^{2}\right) /\left(1+\omega_{\mathrm{pe}}^{2} / \Omega_{\mathrm{e}}^{2}\right),
\end{aligned}
$$

where $\Omega_{j}$ and $\omega_{\mathrm{p} j}$ are the cyclotron frequency and the plasma frequency, respectively, and $j$ refers to ions $(j=i)$ and electrons $(j=\mathrm{e})$. The value of $\omega_{\mathrm{LH}} / \Omega_{\mathrm{i}}$ increases with the plasma density if the magnetic field strength is fixed. In the Large Helical Device (LHD), radio frequency (RF) waves from the plasma are detected $[4,5]$, and RF waves in the frequency range of $\omega_{\mathrm{LH}}$ were observed during the period of perpendicular neutral beam injection (NBI) [6]. Recently, stair-like frequency chirping is observed in the $\mathrm{RF}$ radiation in the frequency range of $\omega_{\mathrm{LH}}$ when the LHD plasma is initiated by almost simultaneous injections of the electron cyclotron wave and the tangential neutral beam [7]. The energetic ions produced by tangential NBI

author'se-mail: toida.mieko@nifs.ac.jp

*) This article is based on the presentation at the 27th International Toki Conference (ITC27) \& the 13th Asia Pacific Plasma Theory Conference (APPTC2018). can have the finite speed across the magnetic field although the angle between the magnetic field and the energetic ion beam is small. The experimental results also show that the riser of the stair is $\Omega_{\mathrm{i}}$ and the RF wave with $\omega \simeq l \Omega_{\mathrm{i}}$, where $l$ is an integer, begins to grow when $\omega_{\mathrm{LH}}$ is close to $l \Omega_{\mathrm{i}}$.

We are studying the excitation mechanism of waves in the frequency range of $\omega_{\mathrm{LH}}$ using a one-dimensional (one spatial coordinate and three velocity components), electromagnetic, particle-in-cell (PIC) code which simulates selfconsistently the full ion and electron dynamics. In ref. [8], we investigated the linear and nonlinear evolution of instabilities due to energetic ions produced by the perpendicular NBI, assuming that the energetic ions have a ring-like distribution in the velocity space perpendicular to the magnetic field. It was shown that in addition to electromagnetic emissions near the ion cyclotron frequency and its harmonics, the high-frequency magnetosonic waves with frequencies slightly smaller than $\omega_{\mathrm{LH}}$ grow to large amplitudes. We also performed the simulations for various plasma densities and demonstrated that the frequency of the large-amplitude magnetosonic wave increases with the electron density when the density is relatively small. The density dependence shown by the simulations qualitatively agrees with the density dependence of RF waves of the lower hybrid wave frequency range observed in the LHD experiments [6]. 
In this paper, we consider a plasma in which the density and $\omega_{\mathrm{LH}}$ are gradually increasing with time, such as at the plasma start-up phase. The density and the value of $\omega_{\mathrm{LH}} / \Omega_{\mathrm{i}}$ are much smaller than those considered in Ref. [8]. In order to study the mechanism of the stair-like frequency chirping observed at the plasma start-up phase of the LHD experiments [7], we perform a simulation of instabilities driven by energetic ions injected continuously in a plasma with the increasing density and the increasing $\omega_{\mathrm{LH}}$. We investigate how the time variation of $\omega_{\mathrm{LH}}$ influences the wave evolution.

\section{Linear Theory}

Before explaining simulations, we look at the linear dispersion relations of energetic ion driven instabilities in the frequency range of $\omega_{\mathrm{LH}}$.

We assume that the velocity distribution function of energetic ions is given by

$$
f\left(v_{\|}, v_{\perp}\right)=\frac{1}{2 \pi u_{\perp}} \delta\left(v_{\|}-u_{\|}\right) \delta\left(v_{\perp}-u_{\perp}\right),
$$

where the subscripts $\|$ and $\perp$ indicate parallel and perpendicular to the external magnetic field, respectively, and $u$ is the initial speed of the energetic ions. We assume that $u_{\perp} / u_{\|} \ll 1$ and $u / v_{\mathrm{A}} \ll 1$, where $v_{\mathrm{A}}$ is the Alfvén speed, because we consider the energetic ions produced by the tangential NBI in a low-density plasma where $v_{\mathrm{A}}$ to be much greater than $u$.

We consider waves propagating perpendicular to the external magnetic field. These waves may play essential roles in the stair-like frequency chirping in the RF radiations observed at the plasma start-up phase in the LHD experiments. The radiations are detected by the antenna located in the direction nearly perpendicular to the magnetic field from the point where the energetic ions are generated by the tangential NBI [7]. Although the excited waves will have both electromagnetic and electrostatic components, we use in this section the electrostatic approximation for simplicity, which may be valid because these waves will have short wavelengths such that $k v_{\mathrm{A}} / \Omega_{\mathrm{i}} \gg 1$ since $u_{\perp} / v_{\mathrm{A}} \ll 1$.

The dispersion relations of the perpendicular electrostatic waves in a plasma consisting of electrons, bulk ions, and the energetic ions can be written as [9]

$$
\epsilon=\frac{\left(1+\omega_{\mathrm{pe}}^{2} / \Omega_{\mathrm{e}}^{2}\right)}{\left(\omega^{2}-\Omega_{\mathrm{i}}^{2}\right)}\left(\omega^{2}-\omega_{\mathrm{LHB}}^{2}\right)+\epsilon_{\mathrm{h}}=0,
$$

where $\epsilon$ is the plasma dielectric function, $\omega$ is the complex frequency, $\omega_{\mathrm{LHB}}$ is the frequency of the Bernstein mode due to the bulk ions near $\omega_{\mathrm{LH}}$, and $\epsilon_{\mathrm{h}}$ is the term due to the energetic ions. Assuming that $k v_{\mathrm{Ti}} / \Omega_{\mathrm{i}} \ll 1$, where $\nu_{\mathrm{Ti}}$ is the bulk-ion thermal speed, and $\omega_{\mathrm{pi}} \gg \Omega_{\mathrm{i}}$, we can approximate $\omega_{\mathrm{LHB}}$ as

$$
\omega_{\mathrm{LHB}}^{2}=\omega_{\mathrm{LH}}^{2}-\frac{\omega_{\mathrm{pi}}^{2}}{\left(1+\omega_{\mathrm{pe}}^{2} / \Omega_{\mathrm{e}}^{2}\right)} \frac{k^{2} v_{\mathrm{Ti}}^{2}}{\Omega_{\mathrm{i}}^{2}},
$$

where electron kinetic effects are neglected. In the limit of $k \rightarrow 0, \omega_{\text {LHB }}$ goes to $\omega_{\text {LH }}$ defined by eq. (1).

The energetic ion term $\epsilon_{\mathrm{h}}$ can be expressed as follows, depending on the magnitude of the wave growth rate $\gamma$. When $\gamma$ is much greater than $\Omega_{\mathrm{i}}$, we can approximate the energetic ions that can interact with the perpendicular waves as the energetic ion beam across the magnetic field. Because this situation is similar to the modified two-stream instabilities $[1,2]$, the energetic ion term $\epsilon_{\mathrm{h}}$ can be written as

$$
\epsilon_{\mathrm{h}}=-\frac{\omega_{\mathrm{ph}}^{2}}{\left(\omega-k_{\perp} u_{\perp}\right)^{2}},
$$

where $\omega_{\text {ph }}$ is the plasma frequency of the energetic ions. The equation (3) with this $\epsilon_{\mathrm{h}}$ indicates that the interaction between the bulk-ion Bernstein mode connecting to $\omega_{\mathrm{LH}}$ and the energetic-ion beam mode $\omega=k_{\perp} u_{\perp}$ can cause strong instabilities [10].

When $\gamma<\Omega_{\mathrm{i}}, \epsilon_{\mathrm{h}}$ is written as $[11,12]$

$$
\epsilon_{\mathrm{h}}=-\sum_{l} \frac{\omega_{\mathrm{ph}}^{2} \lg (\lambda)}{\Omega_{\mathrm{i}}\left(\omega-l \Omega_{\mathrm{i}}\right)}, \quad g(\lambda)=\frac{1}{\lambda} \frac{d J_{l}^{2}(\lambda)}{d \lambda},
$$

where $l$ is an integer, $\lambda=k_{\perp} u_{\perp} / \Omega_{\mathrm{i}}$, and $J_{l}$ is the Bessel function. We consider waves with frequencies near $l \Omega_{\mathrm{i}}$ and write $\omega$ as $\omega=l \Omega_{\mathrm{i}}(1+\Delta)$. Then, the equation (3) leads to

$$
2 l^{2} \Omega_{\mathrm{i}}^{2} \Delta^{2}+\left(l^{2} \Omega_{\mathrm{i}}^{2}-\omega_{\mathrm{LHB}}^{2}\right) \Delta-\frac{\omega_{\mathrm{ph}}^{2}\left(l^{2}-1\right) g}{\left(1+\omega_{\mathrm{pe}}^{2} / \Omega_{\mathrm{e}}^{2}\right)}=0 .
$$

From this, we can obtain the unstable condition for the wave with $\omega \simeq l \Omega_{\mathrm{i}}$ as

$$
\left(l^{2} \Omega_{\mathrm{i}}^{2}-\omega_{\mathrm{LHB}}^{2}\right)^{2}+8 \frac{\omega_{\mathrm{ph}}^{2} \Omega_{\mathrm{i}}^{2} l^{2}\left(l^{2}-1\right) g}{\left(1+\omega_{\mathrm{pe}}^{2} / \Omega_{\mathrm{e}}^{2}\right)}<0 .
$$

This can be satisfied when $l \Omega_{\mathrm{i}} \simeq \omega_{\mathrm{LH}}$ and $g<0$. For $l \geq 2, g$ is negative in the range of $\lambda_{1}<\lambda<\lambda_{2}$, where $\lambda_{1}$ and $\lambda_{2}$ are the points at which $J_{l}^{2}$ becomes maximum and minimum, respectively. Because $\lambda_{1}$ is greater than 1 , we can expect that the wave with $\omega \simeq l \Omega_{\mathrm{i}}$ and $k_{\perp} u_{\perp} / \Omega_{\mathrm{i}}>1$ may be destabilized when $l \Omega_{\mathrm{i}}$ is close to $\omega_{\mathrm{LH}}$. We call this wave the $l$ th harmonic ion cyclotron wave (ICW). The ICWs are supported by the energetic ions. They are not the Bernstein modes due to the bulk ions and can exist in the limit of a cold plasma approximation, $v_{\mathrm{Ti}} \rightarrow 0$.

\section{Simulation Model and Parameters}

We study energetic ion driven instabilities in the frequency range of $\omega_{\mathrm{LH}}$ using a one-dimensional (one spatial coordinate and three velocity components), electromagnetic, particle-in-cell (PIC) code which self-consistently simulates full dynamics of electrons and ions. Although the electrostatic approximation was used in Sec.2, the simulation code treats both the electrostatic and the electromagnetic fields by calculating the full Maxwell's equation. We pay attention to magnetic fluctuations shown in the simulation, as in the previous paper [8]. 
The plasma consists of three components: minority energetic ions, electrons, and bulk ions. The ion-toelectron mass ratio is $m_{\mathrm{i}} / m_{\mathrm{e}}=1000$. The total charge of electrons is equal to that of ions. Initially, the electrons and bulk ions have Maxwell velocity distributions, whereas the energetic ions have a velocity distribution function given by eq. (2).

We set the speed of the energetic ions as $u / v_{\mathrm{A} 0}=0.1$, where $v_{\mathrm{A} 0}$ is the initial Alfvén speed. This is equal to the value of $u / v_{\mathrm{A}}$ for the $\mathrm{H}$ ions with the energy $K=178 \mathrm{keV}$ in the $\mathrm{H}$ plasma with $n_{\mathrm{e}}=0.3 \times 10^{19} \mathrm{~m}^{-3}$ and $B=2.6 \mathrm{~T}$. These values are close to the typical values in the plasma core region at the start-up phase of the LHD plasma [7], where the waves can be excited by energetic ions generated by the tangential NBI. The ratios of $v_{\mathrm{A} 0}$ to the electron thermal speed $v_{\mathrm{Te}}$ is $v_{\mathrm{A} 0} / v_{\mathrm{Te}}=2.6$, which is the same order as that for the electron temperature $T_{\mathrm{e}}=5 \mathrm{keV}$. Assuming that the ion temperature $T_{\mathrm{i}}$ is much smaller than $T_{\mathrm{e}}$, we set $v_{\mathrm{A} 0} / v_{\mathrm{Ti}}=300$.

We choose the angle between the energetic ion beam and the magnetic field as $\alpha=\arctan \left(u_{\perp} / u_{\|}\right)=10^{\circ}$, according to Ref. [7]. Then, because $u_{\perp} / v_{\mathrm{A} 0} \ll 0.1$, we can expect from the linear theory that the energetic ions would excite short-wavelength waves such that $k v_{\mathrm{A}} / \Omega_{\mathrm{i}} \gg 10$. These wavelengths are much smaller than $1 \mathrm{~cm}$, and the variation of the LHD magnetic field and the density for these lengths in the plasma core region would be small. Therefore, we assume that the external magnetic field and the plasma density are uniform.

The simulation system is periodic in the $x$ direction with the length $L_{x}=2048 \Delta_{\mathrm{g}}$, where $\Delta_{\mathrm{g}}$ is the grid spacing, and the electron skin depth is $c / \omega_{\mathrm{pe}}=10 \Delta_{\mathrm{g}}$. The waves propagate in the $x$ direction in the external magnetic field $\boldsymbol{B}_{0}=\left(B_{0} \cos \theta, 0, B_{0} \sin \theta\right)$, where $\theta$ is the propagation angle of the waves. Setting $\theta=89.7^{\circ}$, we simulate the waves propagating nearly perpendicularly to $\boldsymbol{B}_{0}$.

The total number of the simulation particles is $N=$ $4 \times 10^{6}$ at $t=0$. In order to simulate a situation where the energetic ions are injected continuously in a plasma with the increasing density and $\omega_{\mathrm{LH}}$, we increase the numbers of electrons, bulk ions, and energetic ions as the time proceeds. The increase rates of the three species of particles are equal. The positions and gyration phases of newly added particles are given randomly. The newly added electrons and bulk ions obey the Maxwell velocity distributions, whereas the newly added energetic ions obey eq. (2).

Figure 1 shows time variations of the simulation parameters. The electron density $n_{\mathrm{e}}$ normalized by the initial density $n_{\mathrm{e} 0}$, the energetic-ion density $n_{\mathrm{h}}$ normalized by the bulk ion density $n_{\mathrm{i}}$, where $n_{\mathrm{e}}=n_{\mathrm{i}}+n_{\mathrm{h}}$ is satisfied, and the values of $\omega_{\mathrm{LH}} / \Omega_{\mathrm{i}}$ and $\Omega_{\mathrm{e}} / \omega_{\mathrm{pe}}$ are plotted. The increase of $n_{\mathrm{e}} / n_{\mathrm{e} 0}$ causes the decrease of $\Omega_{\mathrm{e}} / \omega_{\mathrm{pe}}$ and the increase of $\omega_{\mathrm{LH}} / \Omega_{\mathrm{i}}$ since $\Omega_{\mathrm{e}}$ and $\Omega_{\mathrm{i}}$ are constant. We study how the increase of $\omega_{\mathrm{LH}} / \Omega_{\mathrm{i}}$ influences wave evolution. The three periods shown by the shaded regions in Fig. 1 will be focused on below.

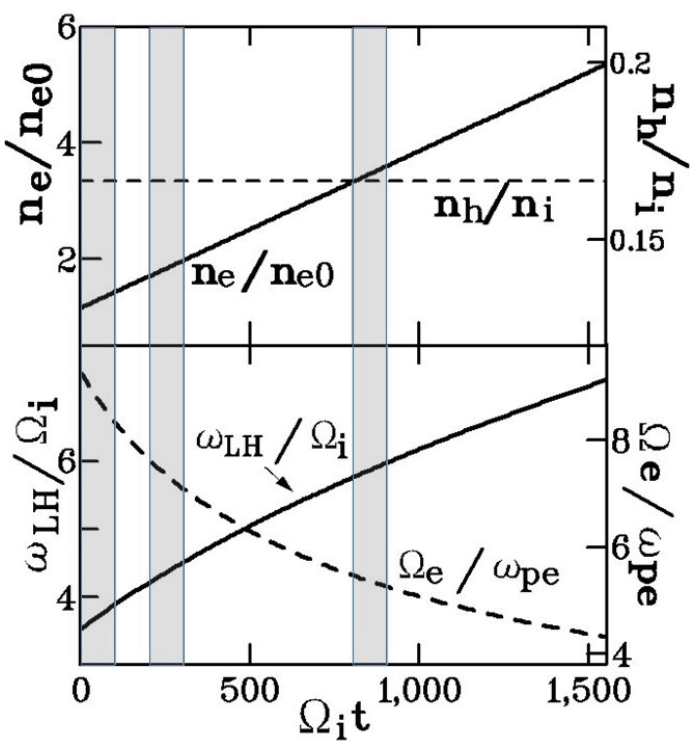

Fig. 1 Time variations of simulation parameters.

\section{Simulation Results}

\subsection{Wave evolution}

We firstly present a result for a plasma without energetic ions. The left panel (a) of Fig. 2 shows the power spectrum $P(k, \omega)$ of magnetic fluctuations obtained for the period from $\Omega_{\mathrm{i}} t=0$ to 100 . During this period, the density and $\omega_{\mathrm{LH}}$ increase from $n_{\mathrm{e}} / n_{\mathrm{e} 0}=1$ to 1.4 and from $\omega_{\mathrm{LH}} / \Omega_{\mathrm{i}}=3.6$ to 3.9 , respectively, as shown by the left shaded region in Fig. 1. The large-amplitude fluctuations near the line $\omega=k v_{\mathrm{A} 0}$ in the long-wavelength region are due to the magnetosonic mode. The fluctuations in the short-wavelength region such that $k v_{\mathrm{A} 0} / \Omega_{\mathrm{i}}>10$ are due to the bulk-ion Bernstein mode that has the dispersion curve connecting to $\omega_{\mathrm{LH}}$, which was expressed as $\omega_{\text {LHB }}$ in Sec. 2.

The right panel (b) of Fig. 2 displays the power spectrum of the magnetic fluctuations in a plasma with the energetic ions for the same period as the left panel (a). Comparison between the two panels shows that the harmonics of ICW with $\omega / \Omega_{\mathrm{i}}=2,3$, and 4 are excited by the energetic ions. Also, the large-amplitude fluctuations are excited near the point $\left(k v_{\mathrm{A} 0} / \Omega_{\mathrm{i}}, \omega / \Omega_{\mathrm{i}}\right)=(280,2.8)$. This point corresponds to the intersection between the curve for the bulk-ion Bernstein mode connecting to $\omega_{\mathrm{LH}}$ and the line $\omega=k_{\perp} u_{\perp}=k_{x} u_{x}$ for the energetic-ion beam mode propagating in the positive $x$ direction, where $u_{x}$ can be estimated as $u_{x} \simeq 0.01 v_{\mathrm{A} 0}$. Here, we neglected $k_{\|}$, because we performed the simulation for the exactly perpendicular case $\theta=90^{\circ}\left(k_{\|}=0\right)$, which is not shown here, and confirmed that the point near which large-amplitude fluctuations are excited when $\theta=90^{\circ}$ is almost the same as the point shown in Fig. 2 (b). As predicted by eqs. (3) and (5), the strong instabilities occurred because of the interaction between the bulk-ion Bernstein mode connecting to $\omega_{\mathrm{LH}}$ and the energetic-ion beam mode.

As the time advances, $\omega_{\mathrm{LH}}$ rises and the power spec- 


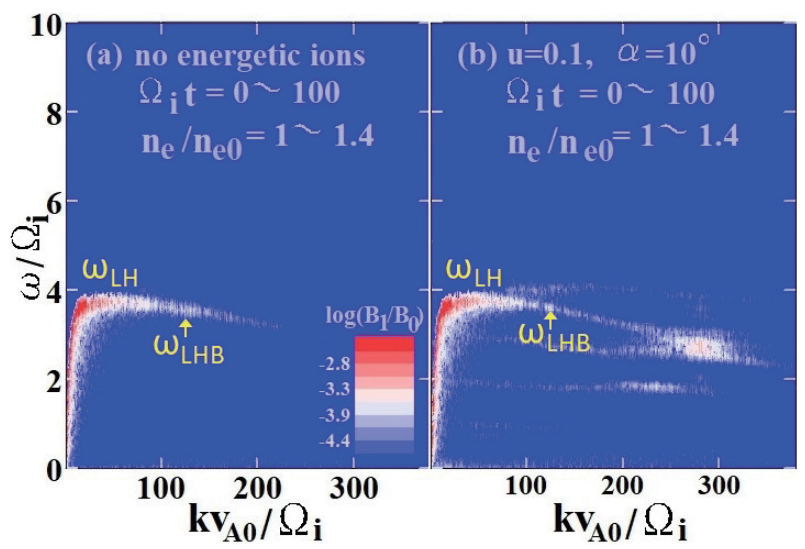

Fig. 2 Power spectra of magnetic fluctuations for the period from $\Omega_{\mathrm{i}} t=0$ to 100 in a plasma without energetic ions (a) and in a plasma with energetic ions (b).

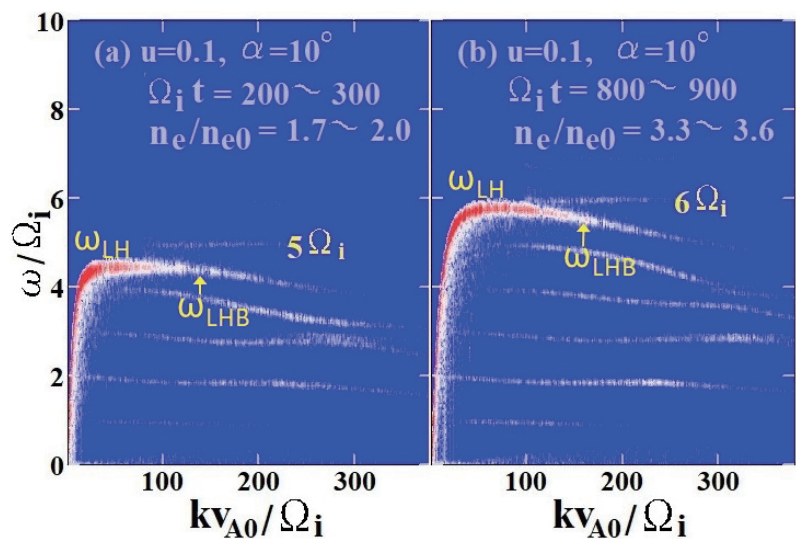

Fig. 3 Power spectra of magnetic fluctuations in a plasma with energetic ions for the periods $200<\Omega_{\mathrm{i}} t<300$ (a) and $800<\Omega_{\mathrm{i}} t<900$ (b).

trum varies. Figure 3 displays the power spectra of magnetic fluctuations for the periods $200<\Omega_{\mathrm{i}} t<300$ (a) and $800<\Omega_{\mathrm{i}} t<900$ (b), which correspond the middle and right shaded regions in Fig. 1, respectively. In the left panel (a), for which $n_{\mathrm{e}} / n_{\mathrm{e} 0}$ and $\omega_{\mathrm{LH}} / \Omega_{\mathrm{i}}$ vary from 1.7 to 2.0 and from 4.2 to 4.5 , respectively, the 5 th harmonic ICW is newly excited slightly above $\omega=\omega_{\text {LH }}$. In the right panel (b), for which $n_{\mathrm{e}} / n_{\mathrm{e} 0}$ and $\omega_{\mathrm{LH}} / \Omega_{\mathrm{i}}$ are in the range of $3.3<n_{\mathrm{e}} / n_{\mathrm{e} 0}<3.6$ and $5.8<\omega_{\mathrm{LH}} / \Omega_{\mathrm{i}}<6.0$, the 6th harmonic ICW appears. The strong instabilities due to the interaction between the bulk-ion Bernstein mode and the energetic ion beam mode are not shown in the two panels of Fig. 3, unlike in the right panel of Fig. 2. This is because the velocity distribution function of the energetic ions is drastically changed by the time $\Omega_{\mathrm{i}} t<100$, which will be shown below.

We now pay attention to how the dispersion curve near $\omega_{\mathrm{LH}}$ varies with time. In the left panel (a) of Fig. 3 where $\omega_{\mathrm{LH}}$ is slightly smaller than $5 \Omega_{\mathrm{i}}$, the frequency of the 5 th harmonic ICW is almost constant, $\omega=5 \Omega_{\mathrm{i}}$, along the horizontal axis $k$ in the wavenumber region $k v_{\mathrm{A} 0} / \Omega_{\mathrm{i}}>100$.

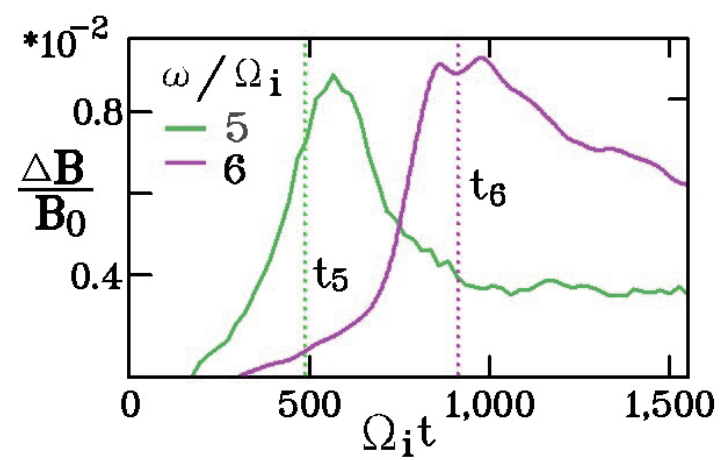

Fig. 4 Time variations of amplitudes of the waves with $\omega \simeq 5 \Omega_{\mathrm{i}}$ and with $\omega \simeq 6 \Omega_{\mathrm{i}}$. These were obtained by integrating $P(k, \omega)$ over the wavenumber region $k v_{\mathrm{A} 0} / \Omega_{\mathrm{i}}>100$.

However, in the right panel (b) of Fig. 3 where $\omega_{\mathrm{LH}}$ is in the range of $5.8<\omega_{\mathrm{LH}} / \Omega_{\mathrm{i}}<6.0$, we cannot see the wave with the constant frequency $\omega=5 \Omega_{\mathrm{i}}$. This is because the 5th harmonic ICW excited by the energetic ions couples with the bulk-ion Bernstein mode that has the dispersion curve connecting to $\omega_{\mathrm{LH}}$. Its frequency gradually decreases from $\omega_{\mathrm{LH}}$ to $5 \Omega_{\mathrm{i}}$. The frequency of the 6th harmonic ICW slightly above $\omega_{\mathrm{LH}}$ is almost constant in this panel. In the power spectrum $P(k, \omega)$ for the next time period, which is not shown here, this wave couples with the bulk-ion Bernstein mode connecting to $\omega_{\mathrm{LH}}$. The 4 th harmonic ICW shown in Fig. 2 (b) couples with the bulk-ion Bernstein mode connecting to $\omega_{\mathrm{LH}}$ in Fig. 3 (a).

In order to show the time variations of amplitudes of waves excited by the energetic ions in more detail, we average the power spectra $P(k, \omega)$ over the wavenumber region $k v_{\mathrm{A} 0} / \Omega_{\mathrm{i}}>100$. We focus on the waves excited by the energetic ions near $\omega=\omega_{\text {LH }}$ and these waves are in this wavenumber region as shown in Fig. 3 . Figure 4 shows the amplitudes of waves with $\omega \simeq 5 \Omega_{\mathrm{i}}$ and $6 \Omega_{\mathrm{i}}$, where the former is in the range of $4.9<\omega / \Omega_{\mathrm{i}}<5.1$, and the latter is in the range of $5.9<\omega / \Omega_{\mathrm{i}}<6.1$. Here, $t_{5}$ and $t_{6}$ are the times when $\omega_{\mathrm{LH}}$ becomes equal to $5 \Omega_{\mathrm{i}}$ and $6 \Omega_{\mathrm{i}}$, respectively. This figure clearly shows that the amplitude of the 5 th harmonic ICW begins to grow near $t=t_{5}$. The amplitude is saturated slightly after $t=t_{5}$ and then gradually decreases, which is caused by the variation of the dispersion curve shown in Fig. 3. The time variation of the wave with $\omega \simeq 6 \Omega_{\mathrm{i}}$ is similar to that with $\omega \simeq 5 \Omega_{\mathrm{i}}$, except that the time $t_{6}$ is important for the evolution of the 6th harmonic ICW.

We then plot the amplitudes of all the waves in the frequency range $0.5<\omega / \Omega_{i}<8$ in Fig. 5, where the color indicates the amplitudes of the magnetic fluctuations and the yellow line represents the frequency $\omega_{\mathrm{LH}}$. We find that the stair-like frequency chirping appears in the region $\omega / \Omega_{\mathrm{i}}>4$. The riser of the stairs is $\Omega_{\mathrm{i}}$ and the wave with $\omega \simeq l \Omega_{\mathrm{i}}$ begin to grow when $\omega_{\mathrm{LH}}$ becomes close to $l \Omega_{\mathrm{i}}$, where $l=5,6$, and 7 . These characteristics are similar to those of the frequency chirping observed in the RF ra- 


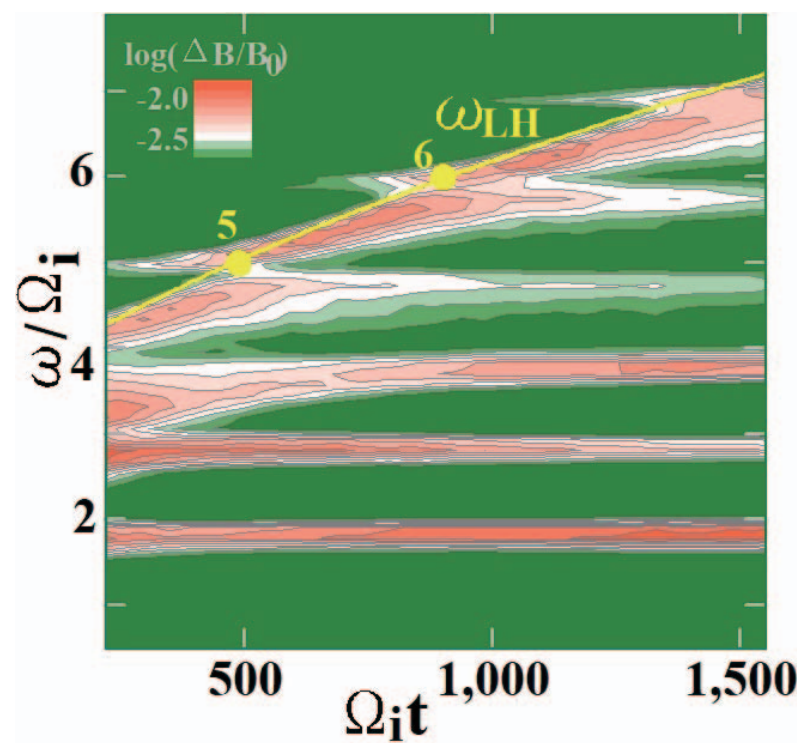

Fig. 5 Time variations of magnetic fluctuations in the frequency range of $0.5<\omega / \Omega_{\mathrm{i}}<8$. The stair-like frequency chirping appears near the line $\omega=\omega_{\mathrm{LH}}$.

diations at the plasma start-up phase of the LHD experiments [7].

\subsection{Velocity distribution function and en- ergy}

Figure 6 displays the time evolution of the velocity distribution functions of the energetic ions. The upper panels (a) and (b) show the perpendicular velocity distribution, and the lower panels (c) and (d) show the parallel velocity distribution. In the panel (a), the perpendicular velocity distribution function $f\left(v_{\perp}\right)$ is drastically changed by the time $\Omega_{\mathrm{i}} t=84$. This is caused by the strong instabilities due to the interaction between the bulk-ion Bernstein mode connecting to $\omega_{\mathrm{LH}}$ and the energetic ion beam mode $\omega=k u_{\perp}$. Because of the change of $f\left(v_{\perp}\right)$, the strong instabilities do not occur after $\Omega_{\mathrm{i}} t=100$, as shown in Fig. 3 . In the panel (b), the steep positive slope, $\partial f\left(v_{\perp}\right) / \partial v_{\perp}>0$, is formed near $v_{\perp} / u_{\perp}=1$ because the energetic ions with $v_{\perp} / u_{\perp}=1$ are added continuously in the simulation system. This can excite ICWs with $\omega=l \Omega_{\mathrm{i}}$ near $\omega_{\mathrm{LH}}$. In the lower panels (c) and (d), the parallel velocity distribution function $f\left(v_{\|}\right)$maintains its shape, although the peak value increases with time. This indicates that effects of the parallel velocity distribution on the wave evolution are negligibly small, compared to those of the perpendicular velocity distribution.

Figure 7 (a) shows the time variation of the kinetic energy of the energetic ions. Here $\Delta K_{\mathrm{h}}$ is defined as

$$
\begin{aligned}
\Delta K_{\mathrm{h}} & =K_{\mathrm{h}}(t)-K_{\mathrm{inj}}(t) \\
& =K_{\mathrm{h}}(t)-N(t) m_{\mathrm{i}}\left(u_{\|}^{2}+u_{\perp}^{2}\right) / 2,
\end{aligned}
$$

where $K_{\mathrm{h}}(t)$ is the total energy of the energetic ions at the time $t, K_{\mathrm{inj}}(t)$ is the total injected energy by the time $t$, and
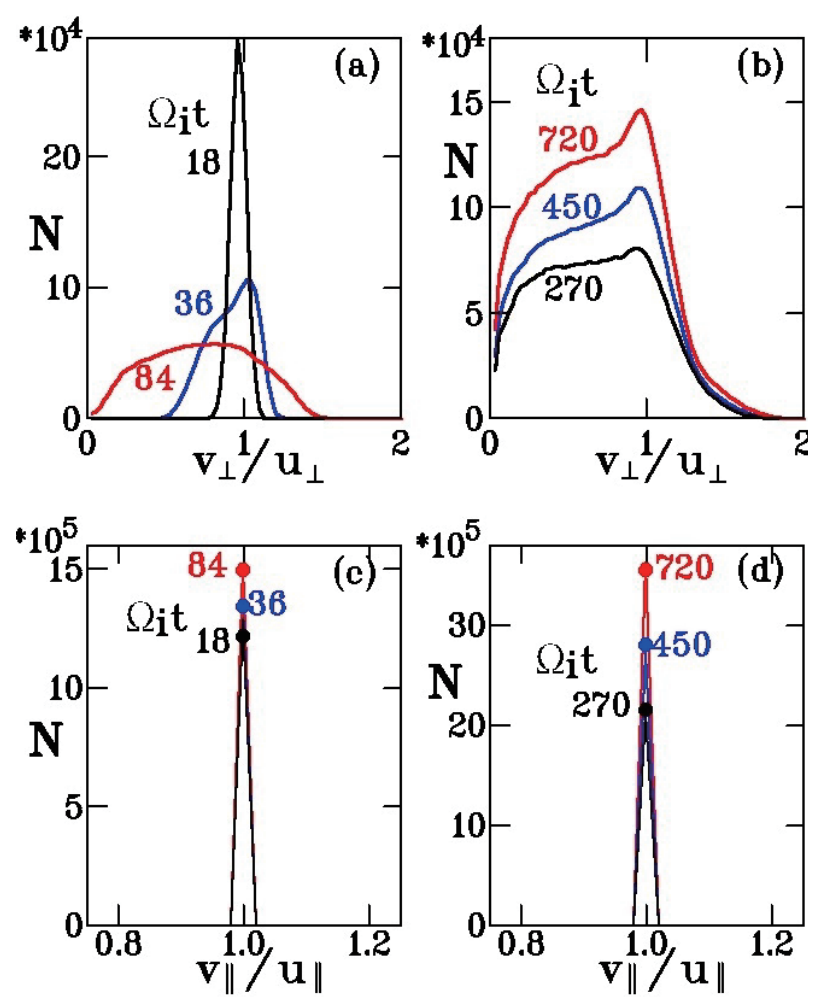

Fig. 6 Time evolution of velocity distribution function of energetic ions. The upper panels (a) and (b) show the pependicular velocity distribution, and the lower panels (c) and (d) show the parallel velocity distribution.

$N(t)$ is the total number of the energetic ions at $t$. The values of $\Delta K_{\mathrm{h}}$ in Fig. 7 (a) are normalized by $K_{\text {inj }}(0)$. The negative $\Delta K_{\mathrm{h}}$ indicates that a part of the injected energy is transferred to the waves. It is clearly shown that $\Delta K_{\mathrm{h}}$ decreases rapidly during the period $0<\Omega_{\mathrm{i}} t<100$ and decreases gradually after $\Omega_{\mathrm{i}} t \simeq 100$. For comparison, we plot the time variations of the energies of magnetic fluctuations in the frequency ranges of $0<\omega / \Omega_{\mathrm{i}}<4.5$ and $4.5<\omega / \Omega_{\mathrm{i}}<7.5$ in Figs. 7 (b) and (c), respectively. The waves in the range of $0<\omega / \Omega_{\mathrm{i}}<4.5$ were excited before $\Omega_{\mathrm{i}} t \simeq 100$, as shown in Fig. 2 (b). From Figs. 7 (b) and (c), we confirm that the waves in the range of $0<\omega / \Omega_{\mathrm{i}}<4.5$ grow rapidly before $\Omega_{\mathrm{i}} t \simeq 100$, whereas the waves in the range of $4.5<\omega / \Omega_{\mathrm{i}}<7.5$ grow gradually after $\Omega_{\mathrm{i}} t \simeq 200$.

In this paper, we assumed that the energetic ions continue to exist in the simulation system after they are injected there. That is, we did not consider the change of $f$ due to the loss of the energetic ions from the system although this may significantly influence the wave evolution. The effect of the energetic ion loss is an important future problem.

\subsection{Different bulk-ion temperature}

In the previous subsections, we presented the results for the case of $v_{\mathrm{A} 0} / v_{\mathrm{Ti}}=300$, where $v_{\mathrm{A} 0}$ is the initial Alfvén speed and $v_{T i}$ is the bulk-ion thermal speed, assuming that the bulk-ion temperature is much lower than the 

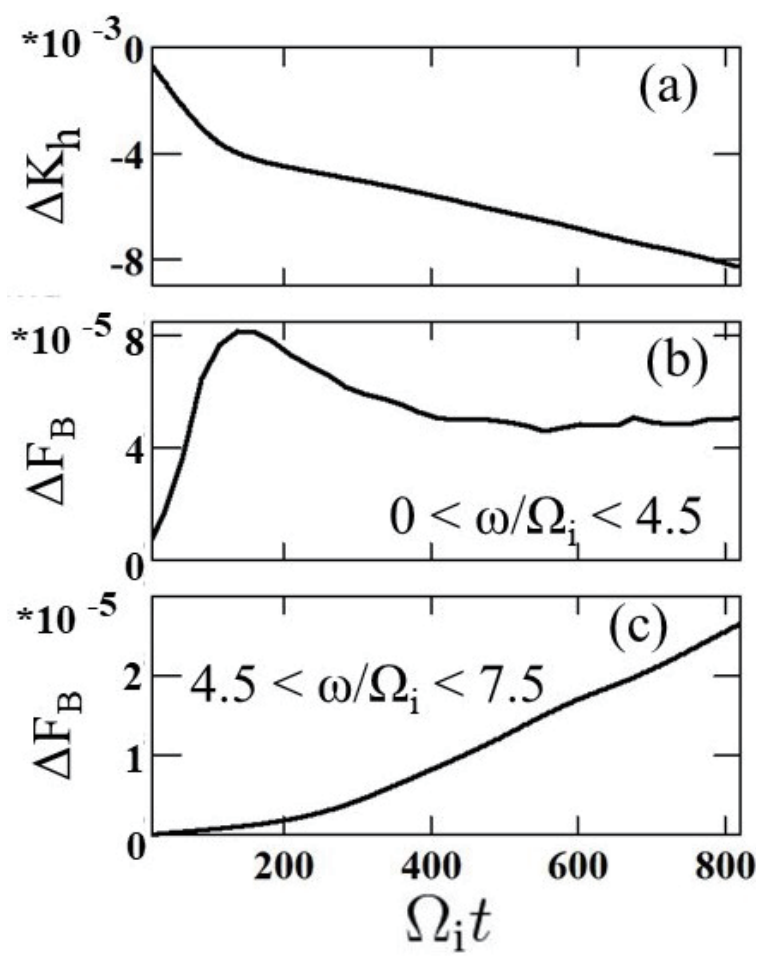

Fig. 7 Time variations of energies of energetic ions (a), magnetic fluctuations in the frequency ranges $0<\omega / \Omega_{\mathrm{i}}<4.5$ (b) and $4.5<\omega / \Omega_{\mathrm{i}}<7.5$ (c). The fluctuation energies are averaged over the wavenumber region $k v_{\mathrm{A} 0}>100$, as in Figs. 4 and 5, and are normalized by $B_{0}^{2} L /(8 \pi)$ with the system length $L$.

electron temperature. We also performed simulations for two cases of different bulk-ion temperatures.

Figure 8 shows the power spectra of magnetic fluctuations for the cases of $v_{\mathrm{A} 0} / v_{\mathrm{Ti}}=2.1 \times 10^{2}$ (a) and $4.2 \times 10^{2}$ (b) obtained for the period from $\Omega_{\mathrm{i}} t=200$ to 300, which is the same period as for Fig. 3 (a). Here, the values of $v_{\mathrm{A} 0}$ and the other parameters are the same as those for Fig. 3 (a). We see almost the same spectra in Figs. 3 (a), 7 (a) and 7 (b), although the values of $v_{\mathrm{Ti}}$ are different. The 5 th harmonic ICW is excited near $\omega_{\mathrm{LH}}$, and the 4th harmonic ICW, which was excited before this period, couples with the bulk-ion Bernstein mode connecting to $\omega_{\mathrm{LH}}$.

Figure 9 shows time variations of amplitudes of the waves with $\omega \simeq 5 \Omega_{\mathrm{i}}$ (a) and with $\omega \simeq 6 \Omega_{\mathrm{i}}$ (b) for the cases of $\left(v_{\mathrm{Ti}} / v_{\mathrm{A} 0}\right) / 10^{2}=4.2,3.0$, and 2.1 , where $t_{5}$ and $t_{6}$ are the same as those in Fig. 4. The waves with $\omega \simeq$ $5 \Omega_{\mathrm{i}}$ and $6 \Omega_{\mathrm{i}}$ have the greatest amplitudes near $t=t_{5}$ and $t_{6}$, respectively, for all the three cases, although there are small differences.

As a result of the wave evolution shown in Figs. 8 and 9 , almost the same chirping in the frequency range of $\omega_{\mathrm{LH}}$ appears in the cases of $v_{\mathrm{A} 0} / v_{\mathrm{Ti}}=4.2 \times 10^{2}$ and $2.1 \times 10^{2}$, which is not shown here. We have not understood the reason for the small differences caused by the effects of the bulk-ion temperature. We will study those effects by performing more simulations in the future.

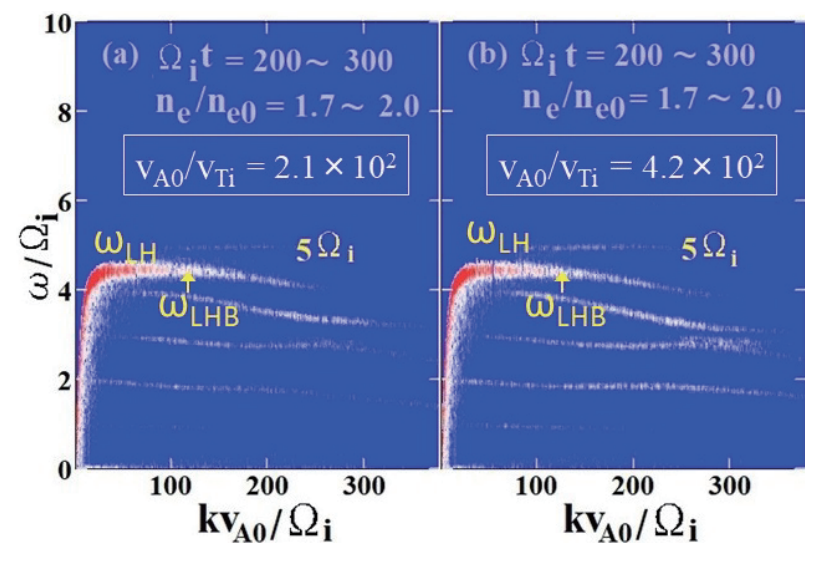

Fig. 8 Power spectra of magnetic fluctuations for the period $200<\Omega_{\mathrm{i}} t<300$ for the cases of $v_{\mathrm{A} 0} / v_{\mathrm{Ti}}=2.1 \times 10^{2}$ (a) and $4.2 \times 10^{2}$ (b).
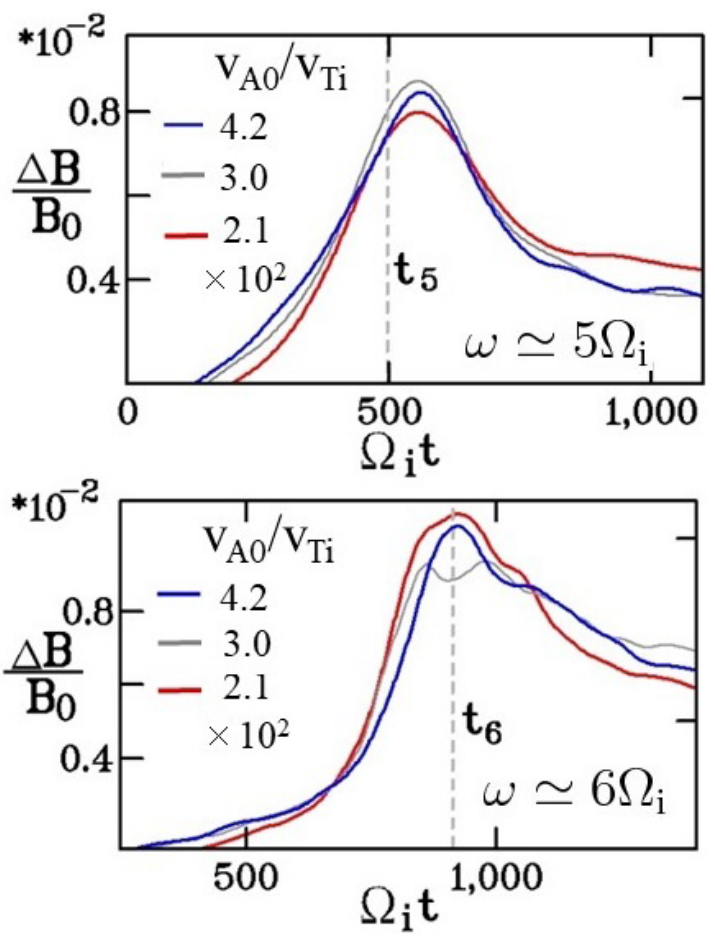

Fig. 9 Time variations of amplitudes of the waves with $\omega \simeq 5 \Omega_{\mathrm{i}}$ (a) and with $\omega_{\mathrm{i}} \simeq 6 \Omega_{\mathrm{i}}$ (b) for the cases of $\left(v_{\mathrm{A} 0} / v_{\mathrm{Ti}}\right) / 10^{2}=$ 4.2, 3.0, and 2.1.

\section{Summary}

By means of a one-dimensional electromagnetic particle code, we have studied instabilities near the lowerhybrid resonance frequency $\omega_{\mathrm{LH}}$ caused by the energetic ions continuously injected in a plasma with the increasing density and $\omega_{\mathrm{LH}}$. We have performed a simulation considering typical parameters for the plasma start-up phase of the LHD experiments. We have analyzed how the increase of $\omega_{\mathrm{LH}}$ influences wave evolution.

It has been shown that the $l$ th harmonic ICW is excited by the energetic ions when $\omega_{\mathrm{LH}}$ becomes close to $l \Omega_{\mathrm{i}}$. This wave couples with the bulk-ion Bernstein mode that 
has the dispersion curve connecting to $\omega_{\mathrm{LH}}$ when $\omega_{\mathrm{LH}}$ becomes greater than $l \Omega_{\mathrm{i}}$. We have also found that as a result of the instabilities driven by the energetic ions and the coupling between the ICWs and the bulk-ion Bernstein mode, the stair-like frequency chirping with the riser $\Omega_{\mathrm{i}}$ appears in the frequency range of $\omega_{\mathrm{LH}}$. The characteristics of the frequency chirping are similar to those of the frequency chirping observed in the RF radiations at the plasma startup phase of the LHD experiments.

In this paper, we did not discuss whether the waves excited by the energetic ions can be detected by the antenna located outside the plasma. This should be studied in the future.

\section{Acknowledgments}

This work is performed with the support and under the auspices of the NIFS Collaboration Research program (NIFS18KNSS103).
[1] J.B. McBride, E. Ott, J.P. Boris and J.H. Orens, Phys. Fluids 15, 2367 (1972).

[2] E. Ott, J.B. McBride, J.H. Orens and J.P. Boris, Phys. Rev. Lett. 28, 88 (1972).

[3] K.A. Akimoto et al., J. Plasma Phys. 34, 445 (1985).

[4] K. Saito et al., Fusion Eng. Des. 84, 1676 (2009).

[5] K. Saito et al., Plasms Sci. Tech. 15, 209 (2013).

[6] K. Saito et al., Plasma Fusion Res. 13, 3402043 (2018).

[7] H. Igami et al., "Observation of stair-like frequency transitions of ion cyclotron harmonic emissions in the lower hybrid frequency range in LHD", presented at International Toki Conference 2018, P1-80.

[8] M. Toida et al., Plasma Fusion Res. 13, 3403015 (2018).

[9] T.H. Stix, Waves in Plasmas (Springer-Verlag, New York, 1992).

[10] D.G. Lominadze, Cyclotron Waves in Plasma (Pergamon Press, 1981).

[11] V.S. Belikov and Ya.I. Kolesnichenko, Sov. Phys. Tech. Phys. 20, 1146 (1976).

[12] R.O. Dendy et al., Phys. Plasmas 1, 3407 (1994). 